\title{
Testing Exponentiality Based on the Lin-Wong Divergence on the Residual Lifetime Data
}

\author{
Mohadeseh Khalili ${ }^{1}$, Arezou Habibirad ${ }^{1}$, and Fatemeh Yousefzadeh ${ }^{2}$ \\ ${ }^{1}$ Department of Statistics, School of Mathematical Sciences, Ferdowsi University of Mash- \\ had, Mashhad, Iran. \\ ${ }^{2}$ Department of Statistics, School of Mathematical Sciences and Statistic, University of Birjand, \\ Birjand, Iran.
}

Received: 12/03/2018, Revision received: 26/12/2019, Published online: 29/08/2019

\begin{abstract}
Testing exponentiality has long been an interesting issue in statistical inferences. The present article is based on a modified measure of distance between two distributions. The proposed new measure is similar to the Kullback-Leibler divergence and it is related to the Lin-Wong divergence applied on the residual lifetime data. A modified measure is developed here which is a consistent test statistic for testing the hypothesis of exponentiality against some alternatives. First, we consider a method similar to Vasicek's and Correa's techniques of estimating the density function in order to construct statistic for LW divergence. Then the critical values of the test are computed, using a Monte-Carlo simulation method. Also, we find the differences of exponential distribution detection power between the proposed test and other tests. It is shown that the proposed test performs better than other tests of exponentiality when the hazard rate is in the form of an increasing function. Finally, a case of application of the proposed test is shown through two illustrative examples.
\end{abstract}

Keywords. Anderson-Darling Statistic, Correa's Technique, Cramer-von Mises Statis-

Mohadeseh Khalili (khalilimohadeseh@gmail.com)

Corresponding Author: Arezou Habibirad (ahabibi@um.ac.ir)

Fatemeh Yousefzadeh (Fyousefzadeh@birjand.ac.ir) 
tic, Exponentiality Test, Goodness of Fit Testing, Kolmogorov-Smirnov Statistic, KullbackLeibler Divergence, Lin-Wong Divergence, Residual Lifetime Data, Vasicek's Technique, Zhang's Statistics.

MSC: 94A17; 62G10.

\section{Introduction}

Recently, many researchers shown an interest in studying the measurement methods of uncertainty associated with probability distribution tests. Of particular interest in probability and statistics is the notion of entropy, introduced by Shannon (1948). If X is a random variable having an absolutely continuous distribution function $G(x)$ with the probability density function (PDF) $g(x)$, then the entropy of the random variable $X$ is defined as

$$
H(g)=-\int_{0}^{\infty} g(x) \log g(x) d x
$$

where the log function denotes the natural logarithm. Some significant applications are divergence measurement and distance information extraction. A prominent source of concern within the applications of probability theory is to find an appropriate measure of the distance between two probability distributions. A number of divergence measures have been already proposed and extended to carry out this task.

Let $g(x)$ and $f(x)$ be PDF of variables $X$ and $Y$, respectively. The Kullback-Leibler (KL) information of $g(x)$ and $f(x)$ has been defined by Kullback and Leibler (1951) based on Shannon entropy as

$$
D_{\mathrm{KL}}(g \| f)=\int_{0}^{\infty} g(x) \log \frac{g(x)}{f(x)} d x .
$$

Various generalized forms of the Shannon entropy and the KL information measure have been introduced over the past 6 decades. We focus on the Lin-Wong (LW) divergence measures in the present paper. The LW divergence is a prominent measure of divergence in this realm. The LW divergence measure is defined by Lin and Wong (1990) as

$$
D_{\mathrm{LW}}(g \| f)=\int_{0}^{\infty} g(x) \log \frac{g(x)}{\frac{1}{2}(g(x)+f(x))} \mathrm{d} x .
$$


Lin and Wong presented and characterized a new direction specific measure, which complements the traditional KL measure. However, they remained closely faithful to the KL divergence. The LW divergence has many desirable properties such as the qualities of nonnegativity, finiteness, semiboundeness, and boundedness (in some cases). The LW divergence is discussed by Lin (1991) and Shioya And Da-Te (1995).

In this case, we observe that a living organism or a subject has survived up to time $\mathrm{t}$, and that the $\mathrm{H}(\mathrm{g})$ entropy measure would not be a useful instrument for measuring the uncertainty about the remaining lifetime of the unit. Consider a system $X$ that has survived up to time $t$. The Equation (1.2) is not appropriate when calculating the uncertainty about the residual life of such a system. Ebrahimi (1996) introduced a new measure to ascertain the uncertainty about the residual life of a random variable $X_{t}=(X \mid X \geq t)$ given by

$$
H\left(g_{t}\right)=-\int_{t}^{\infty} \bar{g}_{t}(x) \log \bar{g}_{t}(x) \mathrm{d} x
$$

in which, $X$ denotes the failure time for a system of living organisms or a component of it and $\bar{g}_{t}(x)=\frac{g(x)}{\bar{G}(t)}$ represents the failure density function, where $G(t)=P(X \leq t)$ is the cumulative distribution function $(\mathrm{CDF})$ and $\bar{G}(t)=1-G(t)$.

Ebrahimi (1996) and Ebrahimi and Pellerey (1995) proposed the concept of residual entropy in terms of a conditional measure. The reader is referred to Nair and Rajesh (1998), Asadi and Ebrahimi (2000), and Navarro et al. (2010) for discussions about the properties and applications of $H\left(g_{t}\right)$.

Furthermore, the KL information based on the Shannon entropy for the residual lifetime data is defined by Ebrahimi and Kirmani (1996a) as follows

$$
D_{\mathrm{KL}}^{(t, \infty)}(g \| f)=\int_{t}^{\infty} \bar{g}_{t}(x) \log \left(\frac{\bar{g}_{t}(x)}{\bar{f}_{t}(x)}\right) \mathrm{d} x .
$$

Most statistical methods, either explicitly or implicitly, presume that the data follow some known distribution pattern in order to derive their results, because there are situations within the context of reliability study, especially within the applied sciences in which it is so desirable to understand if any particular underlying distribution is presented within the data.

In social studies, engineering, medical sciences, reliability studies, and management science, it is very important to know whether the underlying data follow a particular 
distribution. Therefore, many authors are interested in investigating the goodness of fit test methods. Recently, goodness of fit tests based on the Shannon entropy have been developed by many researchers. For example Jager and Wellner (2007), Alizadeh (2010) and Zamanzade and Arghami (2011).

Testing methods to detect exponential distribution patterns still attract much attention and are the topic of a large number of recent researches. Many authors provide test statistics for detecting departures from the hypothesis of exponentiality against specific or general alternatives, for example, see Hanis (1976), Henze and Meintanis (2002b), and Baratpour and Habibirad (2012).

Ebrahimi et al. (1992) obtained an estimator for the exponential goodness of fit test, using the Kullback-Leibler divergence measure on the general. Ebrahimi (1998) performed this estimator, applying (1.5) for the residual lifetime data.

A large number of recent results pertaining to lifetime tests are obtained based on the assumption that the lifetime of a system is described by an exponential distribution. The main focus of this paper is the goodness of fit test for the exponential distribution based on the LW divergence on the residual lifetime data. The exponential data distribution is commonly used in literature and many practical applications to describe sets of data, which are mainly produced through measurements regarding failure times of organisms or man-made systems. The model based on the exponential distribution in such a context is widely known as a lifetime model in the reliability theory. Theoretical justifications of using lifetime model as a reliable probabilistic model for failure times of a system (or its components) are also ubiquitously studied.

In the following, we illustrate the testing exponentiality based on the LW information with the residual lifetime data. In the past, Abbasnejad et al. (2012) improved the applications of LW measure by proposing a new goodness of fit measure to assess the fitness of exponential distributions with data.

The rest of present in the paper is organized as follows. A modified measure of divergence in the context of a residual lifetime is presented in Section 2, and some of its characteristics are introduced. We propose test statistics for exponentiality based on the LW divergence measure to Vasicek's and Correa's techniques when applied to residual lifetime data. In Section 3, behaviors of our test measure are assessed, using some simulated data in terms of value size and distribution detection power. Implementation of the proposed test is illustrated by two examples in Section 4. Finally, our conclusions are summarized within Section 5. 


\section{Test Statistics}

Based on Khalili et al. (2017), using Equation (1.3), the LW divergence on the residual lifetime data can be stated as follows

$$
D_{\mathrm{LW}}^{(t, \infty)}(g \| f)=\int_{t}^{\infty} \bar{g}_{t}(x) \log \frac{\bar{g}_{t}(x)}{\frac{1}{2}\left(\bar{g}_{t}(x)+\bar{f}_{t}(x)\right)} \mathrm{d} x .
$$

A number of implications are explained regarding the definition of divergences.

- It can be readily observed that

$$
\lim _{t \rightarrow \infty} D_{\mathrm{LW}}^{(0, t)}(g \| f)=D_{\mathrm{LW}}(g \| f)=\lim _{t \rightarrow 0^{+}} D_{\mathrm{LW}}^{(t, \infty)}(g \| f),
$$

which is similar to relations pertaining to the KL divergence; see Di Crescenzo and Longobardi (2004) for more details.

- In analogy to the LW discrimination information, in light of assumptions made about $\phi(x)=-\log (x)$, as a convex function, and using the Jensen's inequality, we observe that $D_{\mathrm{LW}}^{(t, \infty)}(g \| f) \geq 0$, and this inequality holds true if and only if $f(x)=g(x)$, for all $x \in(t, \infty)$.

- In analogy to Lin and Wong (1990), we find the upper boundary of the LW divergence value when applied on the residual lifetime data as

$$
D_{\mathrm{LW}}^{(t, \infty)}(g \| f) \leq \log 2
$$

which transforms into $D_{\mathrm{LW}}^{(t, \infty)}(g \| f) \leq 1$, for the logarithm on base of 2 .

Many researchers have introduced the goodness of fit tests based on various entropy estimators. Among those estimators of the entropy, Vasicek's sample entropy measure is most widely used in the goodness of fit tests. Let $X_{1}, X_{2}, \ldots, X_{n}$ be a random sample from a continuous distribution $G$. Using $G(x)=p$, Vasicek (1976) expressed (1.1) as

$$
H(g)=\int_{0}^{1} \log \left(\frac{d}{d p} G^{-1}(p)\right) d p .
$$

After replacing the distribution function $G$ by the empirical distribution function $G_{n}$ and using a difference operator instead of the differential operator, the derivative of 
$G^{-1}(p)$ is estimated by

$$
\frac{X_{(i+m)}-X_{(i-m)}}{\frac{2 m}{n}} .
$$

Therefore $H(g)$ is estimated as

$$
V_{m n}=\frac{1}{n} \sum_{i=1}^{n} \log \left(\frac{n}{2 m}\left(X_{(i+m)}-X_{(i-m)}\right)\right),
$$

where, $m$ is a positive integer with values smaller than $n / 2$, while $X_{(1)} \leq X_{(2)} \leq \cdots \leq X_{(n)}$ are order statistics satisfying the condition $X_{(i)}=X_{(1)}$ if $i<1$, and $X_{(i)}=X_{(n)}$ if $i>n$. Vasicek proved that

$$
V_{m n} \stackrel{p r .}{\rightarrow} H(g) \text { as } n \rightarrow \infty, m \rightarrow \infty, \frac{m}{n} \rightarrow 0 .
$$

Lemma 2.1. Based on Maximum Likelihood Estimator (MLE), the estimator of the parameter $\theta$ in the exponential distribution on residual lifetime data is given by

$$
\hat{\theta}=\frac{1}{\bar{x}-t}
$$

Proof. Since $g_{t}(x)=\frac{\theta e^{-\theta x}}{e^{-\theta t}}$, the proof is clear.

Let $X_{1}, \ldots, X_{n}$ be a random sample from a continuous nonnegative CDF $F(x)$ with a PDF $f(x)$. Consider the following hypothesis:

$$
H_{0}: f(x ; \theta)=f_{0}(x ; \theta),
$$

while the alternative hypothesis would be

$$
H_{1}: f(x ; \theta) \neq f_{0}(x ; \theta) \text {, }
$$

where $f_{0}(x ; \theta)=\theta e^{-\theta x}, x>0$, and $\theta>0$ is unknown.

It motivates us to use $D_{\mathrm{LW}}^{(t, \infty)}\left(f \| f_{0}\right)$ as a test statistic for exponentiality. Note that such an evaluation requires the knowledge of $G$, which is not operational. Instead, we consider a sample estimate of $D_{\mathrm{LW}_{\mathrm{V}}}^{(t, \infty)}\left(f \| f_{0}\right)$. 
The LW divergence on the residual lifetime data, when comparing $f(x)$ against $f_{0}(x)$, is stated as

$$
D_{\mathrm{LW}_{\mathrm{V}}}^{(t, \infty)}\left(f \| f_{0}\right)=\int_{t}^{\infty} \overline{f_{t}}(x) \log \frac{\overline{f_{t}}(x)}{\frac{1}{2}\left(\overline{f_{t}}(x)+\frac{\theta e^{-\theta x}}{e^{-t \theta}}\right)} \mathrm{d} x,
$$

for which the null hypothesis of $D_{\mathrm{LW}}^{(t, \infty)}\left(f \| f_{0}\right)=0$ and positive values of $H_{1}$ are assumed.

To estimate $D_{\mathrm{LW}_{\mathrm{V}}}^{(t, \infty)}\left(f \| f_{0}\right)$, we use the two following methods. In the first method, using $\bar{F}_{t}(x)=P$, similar to Vasicek's method and using difference operator in place of the differential operator, we express (2.2) as

$$
\int_{0}^{1} \log \frac{2\left(-\frac{d F_{t}^{-1}(p)}{d p}\right)^{-1}}{\left(-\frac{d F_{t}^{-1}(p)}{d p}\right)^{-1}+\frac{\theta e^{-\theta x}}{e^{-t \theta}}} d p
$$

There are many types of estimators for $\frac{d F_{t}^{-1}(p)}{d p}$. Amongst various functions proposed for estimating the entropy, Vasicek's sample entropy method has been extensively implemented by scholars when developing statistical procedures based on the entropy concept. We follow Vasicek (1976) regarding the estimator for the density quantile function as follows

$$
\frac{2 m}{n_{0}\left(X_{(i+m)}-X_{(i-m)}\right)} \simeq\left(\frac{d F_{t}^{-1}(p)}{d p}\right)^{-1},
$$

where $X_{(i)}$ is the ordered statistic and the window size $m$ is a positive integer with values smaller than $n / 2$. We obtain an estimator of $D_{\mathrm{LW}}^{(t, \infty)}\left(f \| f_{0}\right)$ as

$$
\hat{D}_{L W_{V}}^{(t, \infty)}\left(f \| f_{0}\right)=-\frac{1}{n_{0}} \sum_{i=1}^{n_{0}} \log \left(\frac{1}{2}+\frac{\left(X_{(i+m)}-X_{(i-m)}\right) n_{0}\left(\hat{\theta} e^{-X_{(i)} \hat{\theta}}\right)}{4 m\left(e^{-t} \hat{\theta}\right)}\right),
$$

where $m$ is a positive integer with values smaller than $\frac{n_{0}}{2}$, while $n_{0}=\# X_{i} \geq t ; t \leq X_{(1)} \leq$ $X_{(2)} \leq \cdots \leq X_{\left(n_{0}\right)}$ are order statistics satisfying the condition $X_{(i)}=X_{(1)}$ if $i<1$, and $X_{(i)}=X_{\left(n_{0}\right)}$ if $i>n_{0}$; and $\hat{\theta}$ is the MLE of $\theta$. 
Now, similar to the proof of Theorem 2 of Alizadeh and Arghami (2011), we prove that the test based on $\hat{D}_{L W_{V}}^{(t, \infty)}\left(f \| f_{0}\right)$ is consistent.

We first consider the estimation of the population entropy $H\left(g_{t}\right)$ by introducing the Vasicek's entropy estimator. Among various entropy estimators, Vasicek's sample entropy is the most widely used in developing statistical procedures.

We use the following theorems in order to obtain a new test statistic.

Theorem 2.1. Let $X_{1}, \ldots, X_{n}$ denote a sample from the continuous distribution with PDF $g(x)$ and CDF $G(x)$, concentrated on $(0,+\infty)$. Then, for any significance level $\alpha \in(0,1)$, the goodness of fit test based on the test statistic $\hat{D}_{L W_{V}}^{(t, \infty)}(g \| f)$ is a consistent test for the hypotheses testing problem.

Proof. According to the consistenty of the empirical distribution function, we have

$$
\frac{2 m}{n\left(X_{(i+m)}-X_{(i-m)}\right)} \stackrel{p}{\rightarrow} \frac{G\left(X_{(i+m)}\right)-G\left(X_{(i-m)}\right)}{\left(X_{(i+m)}-X_{(i-m)}\right)}
$$

also there exists $x_{i} \in\left(X_{(i+m)}, X_{(i-m)}\right)$, in which

$$
\frac{G\left(X_{(i+m)}\right)-G\left(X_{(i-m)}\right)}{\left(X_{(i+m)}-X_{(i-m)}\right)} \simeq g\left(x_{i}\right) .
$$

As $n \rightarrow \infty, m \rightarrow \infty, \frac{m}{n} \rightarrow 0$, then

$$
\frac{1}{n} \sum_{i=1}^{n} \log \left(1+\frac{n f\left(X_{i}\right)\left(X_{(i+m)}-X_{(i-m)}\right)}{2 m}\right) \stackrel{p}{\rightarrow} E_{g}\left(\log \left(1+\frac{f\left(X_{i}\right)}{g\left(X_{i}\right)}\right)\right) .
$$

Hence, using (2.5), the test statistic $\hat{D}_{L W_{V}}^{(t, \infty)}(g \| f)$ is a consistent test.

The next theorem states that the scale of the random variable $X$ does not affect the accuracy of $\hat{D}_{L W_{V}}^{(t, \infty)}(g \| f)$.

Theorem 2.2. Suppose that $X_{1}, \ldots, X_{n}$ is a sequence of iid random variables from a continuous exponential distribution $G(x)$ with a density function $g(x)$ on the residual lifetime data and that $t_{X}$ and $t_{Y}$ are the time points for the random variables $X$ and $Y$, respectively. Put $t_{Y}=k t_{X}$, 
and $Y_{i}=k X_{i}, i=1, \ldots, n$, where $k>0$. Let $\hat{D}_{L W_{V X}}^{\left(t_{X}, \infty\right)}(g \| f)$ and $\hat{D}_{L W_{V Y}}^{\left(t_{Y}, \infty\right)}(g \| f)$ be the estimators for $D_{L W_{V X}}^{\left(t_{x}, \infty\right)}(g \| f)$ and $D_{L W_{V Y}}^{\left(t_{Y}, \infty\right)}(g \| f)$ on the residual lifetime data, respectively. Then, we can see that the test statistic is invariant with respect to scale transformations, and the following properties

(i) $E\left(\hat{D}_{L W_{V X}}^{\left(t_{X}, \infty\right)}(g \| f)\right)=E\left(\hat{D}_{L W_{V Y}}^{\left(t_{Y}, \infty\right)}(g \| f)\right)$,

(ii) $\operatorname{Var}\left(\hat{D}_{L W_{V X}}^{\left(t_{X}, \infty\right)}(g \| f)\right)=\operatorname{Var}\left(\hat{D}_{L W_{V Y}}^{\left(t_{Y}, \infty\right)}(g \| f)\right)$,

(iii) $\operatorname{MSE}\left(\hat{D}_{L W_{V X}}^{\left(t_{X}, \infty\right)}(g \| f)\right)=\operatorname{MSE}\left(\hat{D}_{L W_{V Y}}^{\left(t_{Y}, \infty\right)}(g \| f)\right)$.

Proof. From the right-hand side of (2.1), we have

$$
\hat{\theta}_{Y}=\frac{1}{\bar{Y}-t_{Y}}=\frac{1}{k \bar{X}-k t_{X}}=\frac{1}{k} \hat{\theta}_{X} .
$$

So, we can get $\hat{D}_{L W_{V X}}^{\left(t_{X}, \infty\right)}(g \| f)=\hat{D}_{L W_{V Y}}^{\left(t_{Y}, \infty\right)}(g \| f)$, and the proof is complete.

Lemma 2.2. Similar to $\hat{D}_{L W_{V}}^{(t, \infty)}(g \| f)$ in (2.4), the estimator $\hat{D}_{K L}^{(t, \infty)}(g \| f)$ for the KL divergence on the residual lifetime data is given by Ebrahimi (1998) as

$$
\hat{D}_{K L}^{(t, \infty)}(g \| f)=\frac{1}{n_{0}} \sum_{i=1}^{n_{0}} \log \left(\frac{2 m\left(e^{-t \hat{\theta}}\right)}{\left(X_{(i+m)}-X_{(i-m)}\right) n_{0}\left(\hat{\theta} e^{-X_{(i)} \hat{\theta}}\right)}\right) .
$$

Lemma 2.3. Suppose that $X_{1}, \ldots, X_{n}$ is a random sample from a continuous exponential distribution $G(x)$ with a density function $g(x)$ on residual lifetime data. Then, similar to Theorem 2.1, $D_{K L}^{(t, \infty)}$ is scale invariant and consistent.

(i) $E\left(\hat{D}_{K L_{X}}^{\left(t_{X}, \infty\right)}(g \| f)\right)=E\left(\hat{D}_{K L_{Y}}^{\left(t_{Y}, \infty\right)}(g \| f)\right)$,

(ii) $\operatorname{Var}\left(\hat{D}_{K L_{X}}^{\left(t_{X}, \infty\right)}(g \| f)\right)=\operatorname{Var}\left(\hat{D}_{K L_{Y}}^{\left(t_{Y}, \infty\right)}(g \| f)\right)$,

(iii) $\operatorname{MSE}\left(\hat{D}_{K L_{X}}^{\left(t_{X}, \infty\right)}(g \| f)\right)=\operatorname{MSE}\left(\hat{D}_{K L_{Y}}^{\left(t_{Y}, \infty\right)}(g \| f)\right)$.

Another important way to estimate the LW divergence is to use nonparametric Correa's technique for estimating $\frac{d F^{(-1)}(p)}{d p}$. Correa (1995) proposed a new estimator of 
entropy. This new estimator is based on local linear regression. The Correa estimator of the density function is

$$
b_{i}=\frac{\sum_{j=i-m}^{i+m}\left(X_{(j)}-\bar{X}_{(i)}\right)\left(\frac{j}{n}-\frac{i}{n}\right)}{\sum_{j=i-m}^{i+m}\left(X_{(j)}-\bar{X}_{(i)}\right)^{2}}
$$

where

$$
\bar{X}_{(i)}=\sum_{j=i-m}^{i+m} \frac{X_{(j)}}{2 m+1}
$$

and $m$, is a positive integer, $m<\frac{n}{2}$; and $X_{(1)} \leq X_{(2)} \leq \cdots \leq X_{(n)}$ are the order statistics with $X_{(i)}=X_{(1)}$ if $i<1$, and $X_{(i)}=X_{(n)}$ if $i>n$. Therefore, the LW divergence on the residual lifetime data to Correa's method can be written as

$$
\hat{D}_{L W_{C}}^{(t, \infty)}\left(f \| f_{0}\right)=-\frac{1}{n_{0}} \sum_{i=1}^{n_{0}} \log \left(\frac{1}{2}+\frac{n_{0}\left(\hat{\theta} e^{-X_{(i)} \hat{\theta}}\right)}{2 n b_{i}\left(e^{-t} \hat{\theta}\right)}\right) .
$$

Furthermore, the Kolmogorov-Smirnov (KS) statistic belongs to the supremum class of empirical distribution function (EDF) statistics, and this class of statistics is based on the largest vertical difference between the hypothesized and empirical distributions. Consider $n$ ordered data points, $X_{(1)} \leq X_{(2)} \leq \cdots \leq X_{(n)}$, Conover in 1999 defined the test statistic proposed by Kolmogorov (1933) as

$$
T=\sup _{x}\left|F^{*}(x)-F_{n}(x)\right|
$$

where "sup" stands for supremum, $F^{*}(x)$ is the hypothesized distribution function, and $F_{n}(x)$ is the EDF estimated based on the random sample. So, following the notation of Koziol and Byar (1975), the modifications to the KS statistic for the residual lifetime data may be written as

$$
K S^{(t, \infty)}=\max _{1 \leq i \leq n_{0}}\left\{\frac{i}{n_{0}}-F_{t}^{*}\left(X_{i}\right), F_{t}^{*}\left(X_{i}\right)-\frac{i-1}{n_{0}}\right\},
$$

where $n_{0}=\# X_{i} \geq t$, and

$$
F_{t}^{*}\left(X_{i}\right)=\frac{F\left(x_{i}\right)-F(t)}{\bar{F}(t)}=\frac{\bar{F}(t)-\bar{F}\left(X_{i}\right)}{\bar{F}(t)}=1-\frac{\bar{F}\left(X_{i}\right)}{\bar{F}(t)}=1-\frac{e^{-x_{(i)} \hat{\theta}}}{e^{-t \hat{\theta}}} .
$$


Anderson and Darling (1954) defined the statistic for this test as

$$
W_{n}^{2}=-n-\frac{1}{n} \sum_{i=1}^{n}(2 i-1)\left\{\log F^{*}\left(X_{i}\right)+\log \left(1-F^{*}\left(X_{n+1-i}\right)\right\},\right.
$$

where $F^{*}(x)$ is the cumulative distribution function of the specified distribution, $X_{(i)}$ 's are the ordered data, and $n$ is the sample size. Therefore, the modified Anderson and Darling (AD) statistic for the residual lifetime data can be rewritten as

$$
A D^{(t, \infty)}=-n_{0}-\frac{1}{n_{0}} \sum_{i=1}^{n_{0}}(2 i-1)\left\{\log F_{t}^{*}\left(X_{i}\right)+\log \left(1-F_{t}^{*}\left(X_{n_{0}+1-i}\right)\right\} .\right.
$$

Conover (1999) stated that the Cramer-Von Mises (CVM) test is developed by Cramer (1928), Von Mises (1931), and Kolmogorov (1933). The CVM statistic is as below

$$
C V M=\frac{1}{12 n}+\sum_{i=1}^{n}\left[F^{*}\left(x_{(i)}\right)-\frac{2 i-1}{2 n}\right]^{2}
$$

So, The CVM statistic on the residual lifetime data can be computed as

$$
C V M^{(t, \infty)}=\frac{1}{12 n_{0}}+\sum_{i=1}^{n_{0}}\left[F_{t}^{*}\left(X_{i}\right)-\frac{2 i-1}{2 n_{0}}\right]^{2}
$$

Moreover, Zhang (2002) introduced the new statistics $Z_{A}, Z_{C}$, and $Z_{K}$. The test statistics for residual lifetime data are approximately equivalent to

$$
\begin{gathered}
Z_{A}^{(t, \infty)}=-\sum_{i=1}^{n_{0}}\left[\frac{\log \left\{F_{t}^{*}\left(x_{(i)}\right)\right\}}{n_{0}-i+.5}+\frac{\log \left\{1-F_{t}^{*}\left(x_{(i)}\right)\right\}}{i-.5}\right], \\
Z_{C}^{(t, \infty)}=\sum_{i=1}^{n_{0}}\left[\log \left\{\frac{F_{t}^{*}\left(x_{(i)}\right)^{-1}-1}{\frac{\left(n_{0}-.5\right)}{(i-.75)}-1}\right\}\right]^{2},
\end{gathered}
$$

and

$$
Z_{K}^{(t, \infty)}=\max _{1 \leq i \leq n_{0}}\left\{(i-.5) \log \left[\frac{i-.5}{n_{0} F_{t}^{*}\left(x_{(i)}\right)}\right]+\left(n_{0}-i+.5\right) \log \left[\frac{n_{0}-i+.5}{n_{0}\left\{1-F_{t}^{*}\left(x_{(i)}\right)\right\}}\right]\right\} .
$$




\section{Simulation Study}

A simulation study is performed to analyze the behavior of the proposed test statistic. We determine the critical points, using the Mont-Carlo simulation. For this end, 10000 samples, with different sample sizes $n=5, \ldots, 20$ and 25 (5) 50, cutting points $F(t)=$ $0.25,0.5,0.75$ and significance levels $\alpha=0.1,0.05$, are generated for remaining data which follow the exponential distribution with the mean 1 . Then we compare the quantiles of order $(1-\alpha) \times 100$ for the test. For any choice of $m$, we use the formula $m=[\sqrt{n}+.5]$, which was used by Wieczorkowski and Grzegorzewski (1999).

Tables 1 and 2 give the critical values of $\hat{D}_{L W_{V}}^{(t, \infty)}(g \| f)$ and $\hat{D}_{L W_{C}}^{(t, \infty)}(g \| f)$ for various sample sizes. Note that the test statistics are considered as the basis for comparison, hence the critical values would not depend on the unknown mean parameter.

In order to compare the performance of the proposed test with that of previously published test methods, some alternative methods according to the type of hazard function have been assessed which are listed below

a) Increasing Hazard Rate (IHR) including Gamma: $G(3,1)$, Weibull: $W(3,1)$, and Generalized Exponential: GE(3,1).

b) Decreasing Hazard Rate (DHR) including Gamma: $G(0.5,1)$, Weibull: $W(0.5,1)$, and Generalized-Exponential: $G E(0.5,1)$.

c) Nonmonotone Hazard Rate (NHR) including Log-Normal: $L N(0,0.5)$, Log-Laplace: LL(0,.5), and Generalized-Logistic: GL(0.5,1).

We use 10000 Monte Carlo simulations for $n=10$ and 20 to estimate the statistics $L W_{V}, L W_{C}, K L, A D, C M, K S, Z_{A}, Z_{C}$, and $Z_{K}$ for different cutting points $F(t)=$ $0.25,0.5,0.75$ and significance levels $\alpha=0.1,0.05$.

The simulation results are summarized in Tables 3-8 in terms of the proportions of rejected cases. Tables 3-5 and 6-8 show the results with significance levels of $\alpha=.1$ and $\alpha=.05$, respectively. The values indicated by boldface fonts in each row show that the pertaining statistic has the highest performance when compared to other tests on that row.

As can be seen here, the $L W_{V}$ statistic performs better than other tests for all of the alternatives IHR and the distribution detection power of the $L W_{C}$ and KL tests are 
almost near to that of $L W_{V}$, respectively. Also, the AD statistic has a better performance when the hazard function is in the form of a DHR; while the CM test performs slightly better than AD in a few cases.

Regarding an NHR function, $L W_{V}$ statistic performs better than other tests in some cases; while none of the tests can be considered to have overall advantage over the others. In general, the $L W_{V}$ and $L W_{C}$ statistics are more powerful in detecting the exponential distribution when compared to other statistics in cases within the IHR class, while the AD statistic should be used in the DHR group. Moreover, as the size of the cutting point $F(t)$ grows, the tests including the $L W_{V}$ and $L W_{C}$ tests would be less sensitive to the exponential distributions.

Table 1: Critical values of $D_{L W_{V}}^{(t, \infty)}(g \| f)$ for various $\alpha, n, F(t)$.

\begin{tabular}{|c||c|c|c||c|c|c|}
\hline \multicolumn{1}{|c||}{} & \multicolumn{3}{|c||}{$\alpha=0.1$} & \multicolumn{3}{c|}{$\alpha=0.05$} \\
\hline $\mathrm{n}$ & $\mathrm{F}(\mathrm{t})=0.25$ & $\mathrm{~F}(\mathrm{t})=0.5$ & $\mathrm{~F}(\mathrm{t})=0.75$ & $\mathrm{~F}(\mathrm{t})=0.25$ & $\mathrm{~F}(\mathrm{t})=0.5$ & $\mathrm{~F}(\mathrm{t})=0.75$ \\
\hline 5 & 0.3429 & 0.3424 & 0.3417 & 0.3993 & 0.3906 & 0.3907 \\
6 & 0.2956 & 0.2970 & 0.2909 & 0.3375 & 0.3415 & 0.3435 \\
7 & 0.2810 & 0.2824 & 0.2840 & 0.3249 & 0.3208 & 0.3234 \\
8 & 0.2504 & 0.2467 & 0.2490 & 0.2880 & 0.2884 & 0.2855 \\
9 & 0.2232 & 0.2240 & 0.2254 & 0.2605 & 0.2601 & 0.2559 \\
10 & 0.2031 & 0.2056 & 0.2066 & 0.2335 & 0.2339 & 0.2357 \\
11 & 0.1876 & 0.1876 & 0.1891 & 0.2146 & 0.2174 & 0.2167 \\
12 & 0.1733 & 0.1750 & 0.1741 & 0.2013 & 0.2033 & 0.2019 \\
13 & 0.1708 & 0.1706 & 0.1729 & 0.2026 & 0.1989 & 0.1985 \\
14 & 0.1591 & 0.1580 & 0.1621 & 0.1870 & 0.1860 & 0.1875 \\
15 & 0.1498 & 0.1538 & 0.1520 & 0.1751 & 0.1756 & 0.1735 \\
16 & 0.1425 & 0.1419 & 0.1408 & 0.1663 & 0.1635 & 0.1665 \\
17 & 0.1370 & 0.1361 & 0.1356 & 0.1581 & 0.1568 & 0.1566 \\
18 & 0.1308 & 0.1293 & 0.1281 & 0.1512 & 0.1504 & 0.1512 \\
19 & 0.1228 & 0.1251 & 0.1238 & 0.1431 & 0.1434 & 0.1444 \\
20 & 0.1184 & 0.1180 & 0.1186 & 0.1376 & 0.1357 & 0.1382 \\
25 & 0.1007 & 0.1012 & 0.0995 & 0.1176 & 0.1168 & 0.1172 \\
30 & 0.0855 & 0.0867 & 0.0863 & 0.0998 & 0.1003 & 0.1006 \\
35 & 0.0783 & 0.0775 & 0.0770 & 0.0908 & 0.0897 & 0.0902 \\
40 & 0.0704 & 0.0694 & 0.0697 & 0.0800 & 0.0795 & 0.0820 \\
45 & 0.0636 & 0.0631 & 0.0638 & 0.0748 & 0.0742 & 0.0746 \\
50 & 0.0591 & 0.0589 & 0.0588 & 0.0681 & 0.0687 & 0.0683 \\
\hline
\end{tabular}


Table 2: Critical values of $D_{L W_{C}}^{(t, \infty)}(g \| f)$ for various $\alpha, n, F(t)$.

\begin{tabular}{|c||c|c|c||c|c|c|}
\hline \multicolumn{1}{|c||}{} & \multicolumn{3}{c||}{$\alpha=0.1$} & \multicolumn{3}{|c|}{$\alpha=0.05$} \\
\hline $\mathrm{n}$ & $\mathrm{F}(\mathrm{t})=0.25$ & $\mathrm{~F}(\mathrm{t})=0.5$ & $\mathrm{~F}(\mathrm{t})=0.75$ & $\mathrm{~F}(\mathrm{t})=0.25$ & $\mathrm{~F}(\mathrm{t})=0.5$ & $\mathrm{~F}(\mathrm{t})=0.75$ \\
\hline 5 & 0.6916 & 0.6812 & 0.6819 & 0.7612 & 0.7765 & 0.7675 \\
6 & 0.6164 & 0.6197 & 0.6154 & 0.6965 & 0.6896 & 0.6923 \\
7 & 0.5919 & 0.5897 & 0.5920 & 0.6555 & 0.6594 & 0.6664 \\
8 & 0.5482 & 0.5490 & 0.5515 & 0.6115 & 0.6153 & 0.6047 \\
9 & 0.5183 & 0.5142 & 0.5135 & 0.5677 & 0.5660 & 0.5756 \\
10 & 0.4944 & 0.4894 & 0.4978 & 0.5413 & 0.5439 & 0.5419 \\
11 & 0.4684 & 0.4702 & 0.4718 & 0.5154 & 0.5199 & 0.5201 \\
12 & 0.4553 & 0.4539 & 0.4525 & 0.5011 & 0.4972 & 0.4975 \\
13 & 0.4497 & 0.4508 & 0.4483 & 0.4878 & 0.4878 & 0.4916 \\
14 & 0.4347 & 0.4406 & 0.4383 & 0.4738 & 0.4754 & 0.4728 \\
15 & 0.4264 & 0.4287 & 0.4246 & 0.4615 & 0.4625 & 0.4640 \\
16 & 0.4174 & 0.4138 & 0.4174 & 0.4507 & 0.4495 & 0.4504 \\
17 & 0.4046 & 0.4081 & 0.4075 & 0.4394 & 0.4409 & 0.4391 \\
18 & 0.3997 & 0.3990 & 0.3997 & 0.4335 & 0.4311 & 0.4323 \\
19 & 0.3931 & 0.3921 & 0.3921 & 0.4230 & 0.4225 & 0.4232 \\
20 & 0.3862 & 0.3875 & 0.3880 & 0.4158 & 0.4129 & 0.4201 \\
25 & 0.3695 & 0.3692 & 0.3673 & 0.3917 & 0.3898 & 0.3931 \\
30 & 0.3523 & 0.3528 & 0.3532 & 0.3730 & 0.3728 & 0.3739 \\
35 & 0.3435 & 0.3439 & 0.3447 & 0.3624 & 0.3603 & 0.3602 \\
40 & 0.3334 & 0.3340 & 0.3355 & 0.3513 & 0.3496 & 0.3514 \\
45 & 0.3292 & 0.3301 & 0.3299 & 0.3441 & 0.3458 & 0.3451 \\
50 & 0.3246 & 0.3244 & 0.3237 & 0.3380 & 0.3365 & 0.3376 \\
\hline
\end{tabular}

Table 3: Power comparisons for exponential test, $F(t)=0.25, \alpha=0.1$.

\begin{tabular}{|c|c|c|c|c|c|c|c|c|c|c|}
\hline \multirow[b]{2}{*}{$\mathrm{n}$} & \multirow[b]{2}{*}{ Test } & \multicolumn{3}{|c|}{ IHR } & \multicolumn{3}{|c|}{ DHR } & \multicolumn{3}{|c|}{ NHR } \\
\hline & & $\mathrm{G}(3)$ & $W(3)$ & GE(3) & $G(.5)$ & $\mathrm{W}(.5)$ & GE(.5) & $\mathrm{LN}(.5)$ & $\operatorname{LL}(.5)$ & GL(.5) \\
\hline 10 & $L W_{V}$ & 0.6844 & 0.8699 & 0.473 & 0.0683 & 0.0099 & 0.066 & 0.0809 & 0.1876 & 0.2437 \\
\hline & $L W_{C}$ & 0.6604 & 0.8641 & 0.4562 & 0.0664 & 0.0105 & 0.0649 & 0.0784 & 0.1766 & 0.2377 \\
\hline & KL & 0.5984 & 0.829 & 0.404 & 0.0694 & 0.0483 & 0.0684 & 0.0847 & 0.2231 & 0.2153 \\
\hline & $\mathrm{AD}$ & 0.4684 & 0.7381 & 0.2826 & 0.1361 & 0.4342 & 0.1351 & 0.1603 & 0.3168 & 0.1456 \\
\hline & $\mathrm{CM}$ & 0.5302 & 0.7902 & 0.3322 & 0.1198 & 0.3687 & 0.1157 & 0.1597 & 0.3463 & 0.1761 \\
\hline & KS & 0.456 & 0.689 & 0.2909 & 0.1122 & 0.3369 & 0.113 & 0.1501 & 0.3212 & 0.1574 \\
\hline & $Z_{A}$ & 0.5545 & 0.7459 & 0.3618 & 0.109 & 0.339 & 0.1027 & 0.1404 & 0.3328 & 0.1717 \\
\hline & $Z_{C}$ & 0.5796 & 0.7812 & 0.3766 & 0.1003 & 0.3005 & 0.0982 & 0.131 & 0.3146 & 0.1827 \\
\hline & $Z_{K}$ & 0.4232 & 0.6131 & 0.2718 & 0.1219 & 0.3666 & 0.1211 & 0.1347 & 0.285 & 0.1425 \\
\hline 20 & $L W_{V}$ & 0.877 & 0.9828 & 0.6215 & 0.0444 & 0.0029 & 0.0474 & 0.0553 & 0.1296 & 0.2894 \\
\hline & $L W_{C}$ & 0.835 & 0.974 & 0.5762 & 0.0479 & 0.0046 & 0.0502 & 0.0562 & 0.114 & 0.2663 \\
\hline & KL & 0.8022 & 0.9683 & 0.5313 & 0.0702 & 0.2063 & 0.0719 & 0.1 & 0.2933 & 0.2385 \\
\hline & $\mathrm{AD}$ & 0.8111 & 0.9643 & 0.5199 & 0.1608 & 0.6683 & 0.1489 & 0.2298 & 0.4896 & 0.2053 \\
\hline & CM & 0.8271 & 0.973 & 0.5518 & 0.1399 & 0.6237 & 0.1349 & 0.2333 & 0.5115 & 0.2343 \\
\hline & KS & 0.7531 & 0.9337 & 0.4881 & 0.131 & 0.5814 & 0.1263 & 0.2131 & 0.4786 & 0.2123 \\
\hline & $Z_{A}$ & 0.844 & 0.9585 & 0.5755 & 0.1058 & 0.539 & 0.1019 & 0.2091 & 0.5034 & 0.2294 \\
\hline & $Z_{C}$ & 0.8529 & 0.9605 & 0.5784 & 0.1085 & 0.5221 & 0.1018 & 0.2072 & 0.4875 & 0.2343 \\
\hline & $Z_{K}$ & 0.7404 & 0.8842 & 0.4616 & 0.131 & 0.5819 & 0.1277 & 0.1829 & 0.4275 & 0.1792 \\
\hline
\end{tabular}


Table 4: $\quad$ Power comparisons for exponential test, $F(t)=0.50, \alpha=0.1$.

\begin{tabular}{|c|c|c|c|c|c|c|c|c|c|c|}
\hline & & \multicolumn{3}{|c|}{ IHR } & \multicolumn{3}{|c|}{ DHR } & \multicolumn{3}{|c|}{ NHR } \\
\hline $\mathrm{n}$ & Test & $G(3)$ & $\mathrm{W}(3)$ & GE(3) & $G(.5)$ & W(.5) & GE(.5) & $\mathrm{LN}(.5)$ & LL(.5) & GL(.5) \\
\hline \multirow[t]{9}{*}{10} & $L W_{V}$ & 0.4692 & 0.415 & 0.2515 & 0.076 & 0.0173 & 0.0827 & 0.0532 & 0.102 & 0.2041 \\
\hline & $L W_{C}$ & 0.4601 & 0.4141 & 0.2489 & 0.0735 & 0.0168 & 0.082 & 0.0529 & 0.0998 & 0.2034 \\
\hline & $\mathrm{KL}$ & 0.4136 & 0.3849 & 0.2246 & 0.0801 & 0.0434 & 0.0837 & 0.0622 & 0.1641 & 0.1836 \\
\hline & $\mathrm{AD}$ & 0.289 & 0.2676 & 0.141 & 0.1135 & 0.3489 & 0.1072 & 0.1927 & 0.3052 & 0.1239 \\
\hline & $\mathrm{CM}$ & 0.3407 & 0.3163 & 0.1711 & 0.1047 & 0.298 & 0.0955 & 0.1729 & 0.3164 & 0.1457 \\
\hline & KS & 0.3034 & 0.2812 & 0.1632 & 0.1049 & 0.2788 & 0.1023 & 0.1652 & 0.3068 & 0.1398 \\
\hline & $Z_{A}$ & 0.3569 & 0.2983 & 0.1873 & 0.1018 & 0.2655 & 0.0965 & 0.158 & 0.2965 & 0.1483 \\
\hline & $Z_{C}$ & 0.3776 & 0.3198 & 0.1961 & 0.0962 & 0.2343 & 0.096 & 0.141 & 0.2772 & 0.1598 \\
\hline & $Z_{K}$ & 0.2621 & 0.225 & 0.1461 & 0.107 & 0.2894 & 0.1005 & 0.1596 & 0.2736 & 0.1204 \\
\hline \multirow[t]{9}{*}{20} & $L W_{V}$ & 0.6226 & 0.6013 & 0.2986 & 0.0596 & 0.0042 & 0.0668 & 0.0319 & 0.0545 & 0.2453 \\
\hline & $L W_{C}$ & 0.572 & 0.5768 & 0.2763 & 0.0626 & 0.0064 & 0.0663 & 0.0327 & 0.0501 & 0.2284 \\
\hline & KL & 0.5201 & 0.5269 & 0.2428 & 0.0728 & 0.1297 & 0.0794 & 0.078 & 0.2489 & 0.2012 \\
\hline & $\mathrm{AD}$ & 0.5071 & 0.4656 & 0.2071 & 0.1166 & 0.5302 & 0.1089 & 0.2702 & 0.4683 & 0.166 \\
\hline & $\mathrm{CM}$ & 0.5491 & 0.5136 & 0.2419 & 0.1053 & 0.4932 & 0.0979 & 0.2571 & 0.4816 & 0.1923 \\
\hline & KS & 0.4896 & 0.4467 & 0.2226 & 0.1092 & 0.4582 & 0.0973 & 0.238 & 0.4705 & 0.1836 \\
\hline & $Z_{A}$ & 0.5526 & 0.4821 & 0.2488 & 0.094 & 0.4085 & 0.0936 & 0.22 & 0.4643 & 0.1942 \\
\hline & $Z_{C}$ & 0.5627 & 0.4959 & 0.2477 & 0.0946 & 0.398 & 0.0962 & 0.2177 & 0.4515 & 0.1969 \\
\hline & $Z_{K}$ & 0.4481 & 0.3604 & 0.1954 & 0.1083 & 0.4455 & 0.1079 & 0.2168 & 0.4244 & 0.161 \\
\hline
\end{tabular}

Table 5: Power comparisons for exponential test, $F(t)=0.75, \alpha=0.1$.

\begin{tabular}{|c|c|c|c|c|c|c|c|c|c|c|}
\hline \multirow[b]{2}{*}{$\mathrm{n}$} & \multirow[b]{2}{*}{ Test } & \multicolumn{3}{|c|}{ IHR } & \multicolumn{3}{|c|}{ DHR } & \multicolumn{3}{|c|}{ NHR } \\
\hline & & $\mathrm{G}(3)$ & $W(3)$ & GE(3) & G(.5) & $W(.5)$ & GE(.5) & $\mathrm{LN}(.5)$ & $\operatorname{LL}(.5)$ & GL(.5) \\
\hline \multirow[t]{9}{*}{10} & $L W_{V}$ & 0.2759 & 0.1718 & 0.1522 & 0.088 & 0.0238 & 0.0903 & 0.0456 & 0.0232 & 0.1747 \\
\hline & $L W_{C}$ & 0.2737 & 0.1681 & 0.1493 & 0.0892 & 0.0256 & 0.0915 & 0.0459 & 0.0223 & 0.1727 \\
\hline & KL & 0.2486 & 0.1593 & 0.1443 & 0.0906 & 0.0449 & 0.0913 & 0.0583 & 0.123 & 0.1666 \\
\hline & $\mathrm{AD}$ & 0.1592 & 0.1099 & 0.1066 & 0.1075 & 0.2841 & 0.1011 & 0.2136 & 0.4243 & 0.1178 \\
\hline & CM & 0.1976 & 0.1223 & 0.1177 & 0.1016 & 0.2417 & 0.0988 & 0.1891 & 0.4027 & 0.1337 \\
\hline & KS & 0.1802 & 0.1187 & 0.1158 & 0.0993 & 0.226 & 0.0956 & 0.1771 & 0.3779 & 0.1255 \\
\hline & $Z_{A}$ & 0.1996 & 0.1256 & 0.1236 & 0.1031 & 0.2152 & 0.0975 & 0.1656 & 0.356 & 0.14 \\
\hline & $Z_{C}$ & 0.2149 & 0.1302 & 0.1288 & 0.1008 & 0.1876 & 0.0973 & 0.1449 & 0.324 & 0.1458 \\
\hline & $Z_{K}$ & 0.1542 & 0.1108 & 0.1084 & 0.1035 & 0.2365 & 0.1006 & 0.1761 & 0.3643 & 0.1147 \\
\hline \multirow[t]{9}{*}{20} & $\overline{L W_{V}}$ & 0.3676 & 0.2007 & 0.1606 & 0.0795 & 0.0111 & 0.0843 & 0.0295 & 0.0086 & 0.2116 \\
\hline & $L W_{C}$ & 0.346 & 0.1966 & 0.1552 & 0.0815 & 0.015 & 0.0876 & 0.0316 & 0.0101 & 0.2037 \\
\hline & KL & 0.2993 & 0.1701 & 0.14 & 0.0845 & 0.106 & 0.088 & 0.083 & 0.3133 & 0.1776 \\
\hline & $\mathrm{AD}$ & 0.2374 & 0.1211 & 0.1057 & 0.1024 & 0.4251 & 0.0999 & 0.2829 & 0.6357 & 0.133 \\
\hline & $\mathrm{CM}$ & 0.279 & 0.143 & 0.1215 & 0.1011 & 0.4 & 0.096 & 0.2694 & 0.6326 & 0.1546 \\
\hline & KS & 0.2564 & 0.1429 & 0.1214 & 0.1052 & 0.37 & 0.0996 & 0.2505 & 0.6059 & 0.1495 \\
\hline & $Z_{A}$ & 0.2908 & 0.1452 & 0.1327 & 0.0981 & 0.3322 & 0.0966 & 0.2332 & 0.565 & 0.1682 \\
\hline & $Z_{C}$ & 0.2963 & 0.1496 & 0.1335 & 0.0969 & 0.3207 & 0.0973 & 0.2321 & 0.5602 & 0.1674 \\
\hline & $Z_{K}$ & 0.2204 & 0.1254 & 0.1128 & 0.1036 & 0.3572 & 0.1031 & 0.2405 & 0.5604 & 0.1346 \\
\hline
\end{tabular}


Table 6: Power comparisons for exponential test, $F(t)=0.25, \alpha=0.05$.

\begin{tabular}{|c|c|c|c|c|c|c|c|c|c|c|}
\hline & & \multicolumn{3}{|c|}{ IHR } & \multicolumn{3}{|c|}{ DHR } & \multicolumn{3}{|c|}{ NHR } \\
\hline $\mathrm{n}$ & Test & $\mathrm{G}(3)$ & $W(3)$ & GE(3) & $G(.5)$ & $W(.5)$ & GE(.5) & $\mathrm{LN}(.5)$ & LL(.5) & GL(.5) \\
\hline \multirow[t]{9}{*}{10} & $L W_{V}$ & 0.4925 & 0.7282 & 0.3054 & 0.0287 & 0.0038 & 0.0288 & 0.0352 & 0.1141 & 0.1318 \\
\hline & $L W_{C}$ & 0.4796 & 0.7268 & 0.2995 & 0.0289 & 0.0044 & 0.0296 & 0.0355 & 0.1112 & 0.1302 \\
\hline & KL & 0.4432 & 0.6971 & 0.2719 & 0.0312 & 0.0244 & 0.0347 & 0.0406 & 0.1453 & 0.1245 \\
\hline & $\mathrm{AD}$ & 0.2834 & 0.5472 & 0.1484 & 0.0733 & 0.3565 & 0.0695 & 0.0961 & 0.213 & 0.0707 \\
\hline & $\mathrm{CM}$ & 0.3586 & 0.6235 & 0.2019 & 0.0584 & 0.2927 & 0.0557 & 0.094 & 0.2447 & 0.0937 \\
\hline & $\mathrm{KS}$ & 0.3057 & 0.5273 & 0.1797 & 0.0595 & 0.2652 & 0.054 & 0.0928 & 0.2364 & 0.0901 \\
\hline & $Z_{A}$ & 0.4033 & 0.5998 & 0.236 & 0.0516 & 0.2554 & 0.0505 & 0.0769 & 0.2282 & 0.1012 \\
\hline & $Z_{C}$ & 0.3858 & 0.6104 & 0.2158 & 0.0541 & 0.245 & 0.0503 & 0.0739 & 0.2088 & 0.0982 \\
\hline & $Z_{K}$ & 0.2674 & 0.4355 & 0.1582 & 0.0623 & 0.2882 & 0.0645 & 0.0757 & 0.1945 & 0.0735 \\
\hline \multirow[t]{9}{*}{20} & $L W_{V}$ & 0.7605 & 0.9539 & 0.481 & 0.0196 & 0.0016 & 0.021 & 0.0251 & 0.0878 & 0.1827 \\
\hline & $L W_{C}$ & 0.7064 & 0.9353 & 0.4296 & 0.0209 & 0.0018 & 0.0208 & 0.0247 & 0.0714 & 0.1616 \\
\hline & KL & 0.6718 & 0.9293 & 0.3945 & 0.0322 & 0.1364 & 0.0333 & 0.0516 & 0.2149 & 0.1466 \\
\hline & $\mathrm{AD}$ & 0.6639 & 0.9171 & 0.3648 & 0.095 & 0.5901 & 0.0897 & 0.1507 & 0.3776 & 0.1153 \\
\hline & $\mathrm{CM}$ & 0.702 & 0.9384 & 0.4081 & 0.0829 & 0.5388 & 0.0783 & 0.1524 & 0.4072 & 0.147 \\
\hline & KS & 0.591 & 0.85 & 0.3387 & 0.0728 & 0.4729 & 0.0689 & 0.1326 & 0.3694 & 0.1229 \\
\hline & $Z_{A}$ & 0.7314 & 0.9117 & 0.4495 & 0.0536 & 0.4465 & 0.0512 & 0.1239 & 0.3927 & 0.1445 \\
\hline & $Z_{C}$ & 0.7246 & 0.9102 & 0.4276 & 0.0659 & 0.4631 & 0.0607 & 0.1373 & 0.3888 & 0.1397 \\
\hline & $Z_{K}$ & 0.5736 & 0.7699 & 0.3309 & 0.0761 & 0.4732 & 0.0736 & 0.1007 & 0.3128 & 0.0989 \\
\hline
\end{tabular}

Table 7: Power comparisons for exponential test, $F(t)=0.50, \alpha=0.05$.

\begin{tabular}{|c|c|c|c|c|c|c|c|c|c|c|}
\hline \multirow[b]{2}{*}{$\mathrm{n}$} & \multirow[b]{2}{*}{ Test } & \multicolumn{3}{|c|}{ IHR } & \multicolumn{3}{|c|}{ DHR } & \multicolumn{3}{|c|}{ NHR } \\
\hline & & $\mathrm{G}(3)$ & $W(3)$ & GE(3) & $G(.5)$ & $W(.5)$ & GE(.5) & $\mathrm{LN}(.5)$ & $\operatorname{LL}(.5)$ & GL(.5) \\
\hline \multirow[t]{9}{*}{10} & $L W_{V}$ & 0.3043 & 0.2688 & 0.1387 & 0.0354 & 0.0066 & 0.0351 & 0.0229 & 0.0556 & 0.1122 \\
\hline & $L W_{C}$ & 0.2907 & 0.2652 & 0.1369 & 0.0329 & 0.0065 & 0.0345 & 0.0218 & 0.0524 & 0.1082 \\
\hline & KL & 0.2714 & 0.2514 & 0.1307 & 0.0389 & 0.0185 & 0.036 & 0.0277 & 0.1025 & 0.1073 \\
\hline & $\mathrm{AD}$ & 0.1696 & 0.1501 & 0.0728 & 0.0623 & 0.2736 & 0.0597 & 0.1386 & 0.235 & 0.0613 \\
\hline & $\mathrm{CM}$ & 0.2233 & 0.1993 & 0.0982 & 0.0529 & 0.2254 & 0.0503 & 0.1198 & 0.2428 & 0.079 \\
\hline & KS & 0.1994 & 0.1784 & 0.0904 & 0.0542 & 0.2063 & 0.0492 & 0.1147 & 0.2352 & 0.0808 \\
\hline & $Z_{A}$ & 0.2398 & 0.1925 & 0.108 & 0.0508 & 0.1884 & 0.0477 & 0.099 & 0.2155 & 0.0867 \\
\hline & $Z_{C}$ & 0.2189 & 0.1849 & 0.0978 & 0.0475 & 0.1761 & 0.0458 & 0.0926 & 0.196 & 0.0795 \\
\hline & $Z_{K}$ & 0.1601 & 0.1276 & 0.0732 & 0.0568 & 0.2139 & 0.0541 & 0.1025 & 0.1957 & 0.0631 \\
\hline \multirow[t]{9}{*}{20} & $L W_{V}$ & 0.4774 & 0.4632 & 0.1962 & 0.0284 & 0.0018 & 0.0301 & 0.0114 & 0.0353 & 0.1559 \\
\hline & $L W_{C}$ & 0.4397 & 0.445 & 0.1818 & 0.0291 & 0.0019 & 0.0314 & 0.0135 & 0.0324 & 0.1484 \\
\hline & KL & 0.3999 & 0.4129 & 0.1621 & 0.0392 & 0.0833 & 0.0403 & 0.0429 & 0.1989 & 0.1317 \\
\hline & $\mathrm{AD}$ & 0.3692 & 0.3337 & 0.1254 & 0.066 & 0.454 & 0.0583 & 0.1965 & 0.3937 & 0.0928 \\
\hline & $\mathrm{CM}$ & 0.4114 & 0.3823 & 0.1489 & 0.0585 & 0.407 & 0.0504 & 0.1797 & 0.403 & 0.108 \\
\hline & KS & 0.3455 & 0.3093 & 0.1349 & 0.0563 & 0.3684 & 0.0548 & 0.1597 & 0.3797 & 0.1021 \\
\hline & $Z_{A}$ & 0.414 & 0.3483 & 0.1639 & 0.0426 & 0.3108 & 0.0422 & 0.1398 & 0.3738 & 0.1228 \\
\hline & $Z_{C}$ & 0.3851 & 0.3259 & 0.1374 & 0.0485 & 0.3241 & 0.0463 & 0.1569 & 0.3779 & 0.108 \\
\hline & $Z_{K}$ & 0.3027 & 0.2219 & 0.1122 & 0.0542 & 0.3473 & 0.0557 & 0.1398 & 0.3238 & 0.0876 \\
\hline
\end{tabular}


Table 8: Power comparisons for exponential test, $F(t)=0.75, \alpha=0.05$.

\begin{tabular}{|c|c|c|c|c|c|c|c|c|c|c|}
\hline & & \multicolumn{3}{|c|}{ IHR } & \multicolumn{3}{|c|}{ DHR } & \multicolumn{3}{|c|}{ NHR } \\
\hline $\mathrm{n}$ & Test & G(3) & W(3) & GE(3) & $G(.5)$ & W(.5) & GE(.5) & $\mathrm{LN}(.5)$ & LL(.5) & GL(.5) \\
\hline \multirow[t]{9}{*}{10} & $L W_{V}$ & 0.1636 & 0.0902 & 0.0801 & 0.0428 & 0.0116 & 0.0485 & 0.0203 & 0.0101 & 0.1032 \\
\hline & $L W_{C}$ & 0.1621 & 0.0867 & 0.0786 & 0.0423 & 0.0106 & 0.0462 & 0.0196 & 0.0098 & 0.1012 \\
\hline & $\mathrm{KL}$ & 0.1497 & 0.0867 & 0.0779 & 0.0459 & 0.0188 & 0.0489 & 0.0271 & 0.077 & 0.0966 \\
\hline & $\mathrm{AD}$ & 0.08 & 0.0498 & 0.0486 & 0.0554 & 0.2132 & 0.0528 & 0.1405 & 0.3598 & 0.0578 \\
\hline & CM & 0.1115 & 0.0626 & 0.0592 & 0.0537 & 0.1767 & 0.0516 & 0.1209 & 0.3392 & 0.0722 \\
\hline & KS & 0.0968 & 0.0615 & 0.0552 & 0.0533 & 0.1549 & 0.0493 & 0.1093 & 0.31 & 0.0669 \\
\hline & $Z_{A}$ & 0.1213 & 0.0697 & 0.0689 & 0.0528 & 0.1455 & 0.0482 & 0.1006 & 0.2814 & 0.08 \\
\hline & $Z_{C}$ & 0.1138 & 0.0686 & 0.0656 & 0.0514 & 0.1383 & 0.0478 & 0.095 & 0.2655 & 0.0749 \\
\hline & $Z_{K}$ & 0.0769 & 0.0528 & 0.053 & 0.0585 & 0.1624 & 0.0514 & 0.1075 & 0.2867 & 0.0592 \\
\hline \multirow[t]{9}{*}{20} & $L W_{V}$ & 0.2403 & 0.116 & 0.0944 & 0.0402 & 0.0046 & 0.044 & 0.013 & 0.0047 & 0.124 \\
\hline & $L W_{C}$ & 0.2189 & 0.1112 & 0.0892 & 0.0406 & 0.0049 & 0.0444 & 0.0122 & 0.0052 & 0.1168 \\
\hline & $\mathrm{KL}$ & 0.1831 & 0.0952 & 0.0757 & 0.0413 & 0.0564 & 0.043 & 0.0384 & 0.2461 & 0.0955 \\
\hline & $\mathrm{AD}$ & 0.1507 & 0.065 & 0.0581 & 0.0577 & 0.3605 & 0.0545 & 0.2152 & 0.5882 & 0.0754 \\
\hline & $\mathrm{CM}$ & 0.1857 & 0.0788 & 0.0689 & 0.0546 & 0.3267 & 0.0522 & 0.2021 & 0.5825 & 0.0927 \\
\hline & KS & 0.1583 & 0.0742 & 0.0644 & 0.0541 & 0.2851 & 0.0516 & 0.1751 & 0.537 & 0.082 \\
\hline & $Z_{A}$ & 0.1881 & 0.0861 & 0.0765 & 0.0489 & 0.2447 & 0.0478 & 0.1454 & 0.4889 & 0.0985 \\
\hline & $Z_{C}$ & 0.1756 & 0.0789 & 0.0707 & 0.0548 & 0.2634 & 0.0512 & 0.1637 & 0.5049 & 0.0915 \\
\hline & $Z_{K}$ & 0.1192 & 0.0594 & 0.0539 & 0.0482 & 0.2551 & 0.0517 & 0.1469 & 0.477 & 0.0692 \\
\hline
\end{tabular}

\section{Illustrative Examples}

In this section, we consider the proposed goodness of fit test with real data. We present two examples to illustrate the use of test $\hat{D}_{L W_{V}}^{(t, \infty)}(g \| f)$ for testing the validity of exponential distribution.

Examples 4.1. The data given below consist of the amount of lifetimes for 21 tools from Meaker (1987)(see Table 9).

A residual truncated reminder sample is obtained in terms of $t$ value which is determined from the $F(t)=0.25$ based on the real sample. To do so, we have obtained the truncated point $t=45.6$ equivalent to quantile (0.25). So 6 out of 21 observations are eliminated while 15 data points kept for the study. In this way, we can examine the critical value of $\alpha=0.1,0.05$ in Table 1 and $n_{0}=15$, using the sample p-value and the LW statistic value for our sample.

In the same way, we obtain $t=55.56$ for $F(t)=0.50$. So, 11 members of the data are eliminated and $n_{0}=10$ of the data are kept. Also, for $F(t)=0.75$, we compute $t=93.12$. In that case, 16 members of the data are omitted and $n_{0}=5$ of the data are kept. Table 10 shows critical values, test statistics, and p-values. Since the values of 
$\hat{D}_{L W_{V}}^{(t, \infty)}(g \| f)$ are less than the critical values (or $\mathrm{p}$-values are larger than $\alpha$ ), the test accepts the null hypothesis that data follow from an exponential distribution at significance levels $\alpha=0.1$ and $\alpha=0.05$.

Table 9: Amount of lifetime for 21 tools in Example 4.1.

\begin{tabular}{|ccccccccccc|}
\hline 17.88 & 28.92 & 23 & 41.52 & 42.12 & 45.6 & 48.4 & 51.84 & 51.96 & 54.12 & 55.56 \\
67.8 & 68.64 & 68.88 & 84.12 & 93.12 & 98.64 & 105.84 & 127.92 & 128.04 & 173.4 & \\
\hline
\end{tabular}

Table 10: Critical values, test statistics, and p-values in Example 4.1.

\begin{tabular}{|c|c|c|c|c|}
\hline $\mathrm{p}$-value & $D_{\mathrm{LW}}^{(t, \infty)}(g \| f)$ & Critical value & Significance level & $\mathrm{F}(\mathrm{t})$ \\
\hline \hline 0.4946 & 0.0771 & 0.1498 & $\alpha=0.1$ & 0.25 \\
0.4946 & 0.0771 & 0.1751 & $\alpha=0.05$ & \\
\hline \hline 0.1629 & 0.1769 & 0.2056 & $\alpha=0.1$ & 0.50 \\
0.1629 & 0.1769 & 0.2339 & $\alpha=0.05$ & \\
\hline \hline 0.5649 & 0.1571 & 0.3417 & $\alpha=0.1$ & 0.75 \\
0.5649 & 0.1571 & 0.3907 & $\alpha=0.05$ & \\
\hline
\end{tabular}

Examples 4.2. The normal distribution is considered in this example. Table 11 shows yearly amounts of rainfall in the New York city from 1910 to 1973 (Parzen (1979)).

In this case, we have $n=63, n_{0}=47, t=61.95$, equivalently $F(61.95)=0.25$. Similarly, we compute $t=79.3$ for $F(t)=0.50$. Therefore, 32 data are omitted and $n_{0}=31$ data kept. Also, we obtain $t=93.95$ for $F(t)=0.75$. That is, 47 data are eliminated and $n_{0}=16$ data kept. The result of the goodness of fit test for the exponential distribution is presented in Table 12.

Table 12 shows critical values, test statistics, and p-values. Since the values of $\hat{D}_{L W_{V}}^{(t, \infty)}(g \| f)$ are greater than the critical values (or $\mathrm{p}$-values are smaller than $\alpha$ ), the data do not follow from the exponential distribution at the significance levels $\alpha=0.1$ and $\alpha=0.05$. 
Table 11: Yearly rain precipitation in the New York city in Example 4.2.

\begin{tabular}{|ccccccccccc|}
\hline 12.5 & 25.0 & 39.8 & 39.9 & 40.1 & 46.7 & 49.1 & 49.6 & 51.1 & 51.6 & 53.5 \\
54.7 & 55.5 & 55.9 & 58.0 & 60.3 & 63.6 & 65.4 & 66.1 & 69.3 & 70.9 & 71.4 \\
71.5 & 71.8 & 72.9 & 74.4 & 76.2 & 77.8 & 78.1 & 78.4 & 79.0 & 79.3 & 79.6 \\
80.7 & 82.4 & 82.4 & 83.0 & 83.6 & 83.6 & 84.8 & 85.5 & 87.4 & 88.7 & 89.6 \\
89.8 & 89.9 & 90.9 & 97.0 & 98.3 & 101.4 & 102.4 & 103.9 & 104.5 & 105.2 & 110.0 \\
110.5 & 110.5 & 113.7 & 114.5 & 115.6 & 120.7 & 124.7 & 126.4 & & & \\
\hline
\end{tabular}

Table 12: Critical values, test statistics, and p-values in Example 4.2.

\begin{tabular}{|c|c|c|c|c|}
\hline p-value & $D_{\mathrm{LW}_{\mathrm{V}}}^{(t, \infty)}(g \| f)$ & Critical value & Significance level & $\mathrm{F}(\mathrm{t})$ \\
\hline \hline 0.0000 & 0.1406 & 0.0615 & $\alpha=0.1$ & 0.25 \\
0.0000 & 0.1406 & 0.0717 & $\alpha=0.05$ & \\
\hline \hline 0.0000 & 0.1017 & 0.0860 & $\alpha=0.1$ & 0.50 \\
0.0000 & 0.1017 & 0.1001 & $\alpha=0.05$ & \\
\hline \hline 0.0000 & 0.2215 & 0.1408 & $\alpha=0.1$ & 0.75 \\
0.0000 & 0.2215 & 0.1665 & $\alpha=0.05$ & \\
\hline
\end{tabular}

\section{Conclusions}

In this article, the LW divergence on the general data is modified and applied on the residual lifetime data. Furthermore, in the present study, we construct a consistent goodness of fit test for the exponential distribution on the LW divergence measure on the residual lifetime data. By a simulation study, the powers of the proposed tests are computed under several alternatives and different sample sizes. It is shown that $L W_{V}$ and $L W_{C}$ tests on the residual lifetime data are better than other competing tests in the IHR function.

It may be noted that the LW divergence on the residual lifetime data, can be used to construct general goodness of fit tests (not just for exponentiality). One can consider any known density function (with known or unknown parameters) under the null 
hypothesis and use it instead of the function $f(x)$ in the definition of LW distance to obtain the test statistic. For example, a test of normality is considered by the authors; however, it has a poor performance, but it can be extended for the other distributions such as Weibull, gamma, and lognormal in the future. Also, a work is under way by us for testing exponentiality based on the LW divergence on the past lifetime data.

\section{Acknowledgement}

We would like to thank the referees and the associate editor for constructive suggestions.

\section{References}

Abbasnejad, M., Arghami, N. R., and Tavakoli, M. (2012), A goodness of fit test for exponentiality based on Lin Wong information. JIRSS. 11(2), 191-202.

Alizadeh, N. H. (2010), A new estimator of entropy and its application in testing normality. Journal of Statistical Computation and Simulation. 80(4), 1151-1162.

Alizadeh N. H. and Arghami, N. R. (2011b), Testing exponentiality based on characterizations of the exponential distribution. Journal of Statistical Computation and Simulation. 81(11), 1641-1651.

Anderson, T. W. and Darling, D. A. (1954), A test of goodness of fit. Journal of American Statistical Association. 49, 765-769.

Asadi, M. and Ebrahimi, N. (2000), Residual entropy and its characterizations in terms of hazard function and mean residual life function. Statistics and probability letters. 49(3), 263-269.

Baratpour, S. and Habibirad, A. (2012), Testing goodness-of-fit for exponential distribution based on cumulative residual entropy. Communications in Statistics-Theory and Methods. 41(8), 1387-1396.

Choi, B., Kim, K., and Song, S. H. (2004), Goodness-of-Fit Test for Exponentiality Based on Kullback-Leibler Information. Communications in Statistics-Simulation and Computation. 33(2), 525-536.

Conover, W. J. (1999), Practical Nonparametric Statistics. John Wiley and Sons, New York. 
Correa, J. C. (1995), A new estimator of entropy. Communications in Statistics-Theory and Methods. 24(10), 2439-2449.

Cramer H. (1928), On the composition of elementary errors, Skandinavisk Aktuarietidskrift. 11:13-74, 141-180.

Di Crescenzo, A. and Longobardi, M. (2004), A measure of discrimination between past lifetime distributions. Statistics and Probability Letters. 67(2), 173-182.

Ebrahimi, N., Habibullah, M., and Soofi, E. S. (1992), Testing exponentiality based on Kullback-Leibler information. Journal of the Royal Statistical Society. Series B (Methodological). 739-748.

Ebrahimi, N. (1996), How to measure uncertainty about residual lifetime. Sankhya- $A$. 58(1), 48-57.

Ebrahimi, N. (1998), Testing exponentiality of the residual life, based on dynamic Kullback-Leibler information. IEEE transactions on reliability. 47(2), 197-201.

Ebrahimi, N. and Kirmani, S. N. U. A. (1996a), A measure of discrimination between two residual life-time distributions and its applications. Annals of the Institute of Statistical Mathematics. 48(2), 257-265.

Ebrahimi, N. and Pellerey, F. (1995), New partial ordering of survival functions based on the notion of uncertainty. Journal of Applied Probability. 32(1), 202-211.

Gurevich, G. and Davidson, A. (2008), Standardized forms of Kullback-Leibler information based statistics for normality and exponentiality. Computer Modelling and New Technologies. 12(1), 14-25.

Hanis, C. M. (1976), A Note on Testing for Exponentiality. Num. Res. Bogist. 28(3), 169-176.

Henze, N. and Meintanis, S. G. (2002b), Goodness-of-fit tests based on a new characterization of the exponential distribution. Communications in Statistics, Theory and Methods. 31(9), 1479-1497.

Jager, L. and Wellner, J. A. (2007), Goodness-of-fit tests via phi-divergences. The Annals of Statistics. 2018-2053.

Khalili, M., Habibirad, A., and Yousefzadeh, F. (2017), Some Properties of Lin Wong Divergence on the Past Lifetime Data. Communications in Statistics, Theory and Methods. 3464-3476. 
Kolmogorov, A. N. (1933), Sulla determinazione empirica di una legge di distribuzione. Giornale dell Instituto Italiano degli Attuari. 4, 83-91.

Koziol, J. A. and Byar, D. P. (1975), Percentage points of the asymptotic distributions of one and two sample KS statistics for truncated or censored data. Technometrics. 17(4), 507-510.

Kullback, S. and Leibler, R. A. (1951), On information and sufficiency. The annals of mathematical statistics. 22(1), 79-86.

Lin, J. (1991), Divergence measures based on the Shannon entropy. IEEE Transactions on Information theory. 37(1), 145-151.

Lin, J. and Wong, S. K. M. (1990), A New Directed Divergence Measure and Its Characterization. International Journal Of General System. 17(1), 73-81.

Meaker, M. K. (1987), Clinical Gerontology: A Guide to Assessment and Intervention. American Journal of Occupational Therapy. 41(4), 269-269.

Nair, K. R. M. and Rajesh, G. (1998), Characterization of the probability distributions using the residual entropy function. Journal of the Indian Statistics Association. 36, 157-166.

Navarro, J., Del Aguila, Y., and Asadi, M. (2010), Some new results on the cumulative residual entropy. Journal of Statistical Planning and Inference. 140(1), 310-322.

Navarro, J., Franco, M., and Ruiz, J. M. (1998), Characterization through moments of the residual life and conditional spacings. Sankhya, The Indian Journal of Statistics, Series A. 36-48.

Parzen, E. (1979), Nonparametric Statistical Data Modeling. Journal of the American Statistical Association, 74(365), 105-121.

Shannon, C. E. (1948), A mathematical theory of communication. Bell System Technical Journal. 27, 379-423.

Shioya, H. and Da-Te, T. (1995), A generalization of Lin divergence and the derivation of a new information divergence. Electronics and Communications in Japan (Part III: Fundamental Electronic Science). 78(7), 34-40.

Vasicek, O. (1976), A test for normality based on sample entropy. Journal of Research Statistical Society. 38(Serial. B), 54-59. 
Von Mises, R. (1931), Wahrscheinlichkeitsrechnung und Ihre Anwendung in der Statistik und Theoretischen Physik. F. Deuticke, Leipzig. 6(1).

Wieczorkowski, R. and Grzegorzewski, P. (1999), Entropy estimators-Improvements and comparisons. Communication Statistics Computing and Simulation. 28, 541-567.

Zamanzade, E. and Arghami, N. R. (2011), Goodness of fit test based on correcting moments of modified entropy estimator.Journal of Statistical Computation and Simulation. 81, 2077-2093.

Zhang, J. (2002), Powerful goodness of fit tests based on the likelihood ratio. Journal of the Royal Statistical Society: Series B (Statistical Methodology). 64(2), 281-294. 
